\title{
A Hyperbolic Lindstedt-Poincaré Method for Homoclinic Motion of a Kind of Strongly Nonlinear Autonomous Oscillators
}

\author{
Y. Y. Chen • S. H. Chen • K. Y. Sze
}

\begin{abstract}
A hyperbolic Lindstedt-Poincaré method is presented to determine the homoclinic solutions of a kind of nonlinear oscillators, in which critical value of the homoclinic bifurcation parameter can be determined. The generalized Liénard oscillator is studied in detail, and the present method's predictions are compared with those of Runge-Kutta method to illustrate its accuracy.
\end{abstract}

Keywords Lindstedt-Poincaré Method, Hyperbolic function, Nonlinear autonomous oscillator, Homoclinic orbit

\section{Introduction}

In the last few decades, many new techniques have been presented for obtaining periodic solution of the nonlinear oscillator equation in the form of

$$
g(x)=\varepsilon f(x, x)
$$

The project supported by the National Natural Science Foundation of China (10672193),

Sun Yat-sen University (Fu Lan Scholarship) and the University of Hong Kong (CRGC grant).

Y. Y. Chen • S. H. Chen $(\square)$

Department of Applied Mechanics and Engineering, Sun Yat-sen University, 510275, Guangzhou, China email: stscsh@mail.sysu.edu.cn
Y. Y. Chen • K. Y. Sze

Department of Mechanical Engineering, The University of Hong Kong, Pokfulam, Hong Kong SAR, China where $g(x)$ and $f(x, x)$ are nonlinear functions of their arguments and $\varepsilon$ is a small positive parameter. With reference to the periodic functions employed in the solutions, these techniques can be categorized into the circular (trigonometric) function perturbation procedures, the elliptic function perturbation procedures and the generalized harmonic function perturbation procedures as described in [1]. Furthermore, much effort has been paid to investigate the stability and bifurcation of periodic solution. For example,Wang and $\mathrm{Hu}[2]$ presented a modified averaging scheme with application to the secondary Hopf bifurcation of a delayed van der Pol oscillator. Gan and $\mathrm{He}$ [3] studied the structural safety in a kind of excited Duffing oscillator.

In most cases, the homoclinic (heteroclinic) orbit is a separatrix of the periodic solutions and the non-periodic solutions of a nonlinear dynamic system, and thus plays an important role in studying the global bifurcation of nonlinear systems and attracts considerable attention. Xu et al. [4] presented a perturbation-incremental method to study the separatrices and the limit cycles of strongly nonlinear oscillators, which was used by Chan et al. [5] and Chen et al. [6] to study the stability and the bifurcations of limit cycles and the semi-stable limit cycles, respectively. Moreover, Zhang and Lu [7] presented a frequency-incremental method to study the homoclinic bifurcation of strongly nonlinear 
oscillators, and Zhang et al. [8] applied the undetermined fundamental frequency method to predict the heteroclinic bifurcation of strongly nonlinear oscillators. Belhaq [9] and his coworkers presented an analytical method to predict homoclinic bifurcation of autonomous oscillators, in which the homoclinic bifurcation value was determined by considering the period of the limit cycles which approaches infinity. To improve the accuracy of their technique, they also employed the elliptic averaging method [10] and the elliptic Linstedts-Poincaré method [11] which lead to the same results as the standard Melnikov method. It isworth noting that the afore-mentioned techniques are based on the periodic functions and, thus, cannot yield the homoclinic (heteroclinic) solution. However, the present authors have recently proposed a hyperbolic perturbation method to determine homoclinic orbits of some strongly nonlinear autonomous oscillators [1].

Based on the previous work, a hyperbolic Lindstedt-Poincaré method is presented in this paper to determine the homoclinic orbits of certain nonlinear autonomous oscillators, in which hyperbolic functions, rather than the usual periodic functions, are employed in the classical Lindstedt-Poincaré procedure and the critical value of the homoclinic bifurcation parameter $\mu$ can be thereby determined. To show the essences of the present method, the typical generalized

Liénard equation is studied in detail, and comparison is made between the results predicted by Runge-Kutta method and the present method. It can be seen that the present method attains fairly good accuracy even for amoderately large value of $\varepsilon$.

\section{The hyperbolic Lindstedt-Poincaré method}

To demonstrate the hyperbolic Lindstedt-Poincaré method, the following nonlinear autonomous system is considered:

$$
c_{1} x+c_{2} x^{2}=\varepsilon f(\mu, x, x)
$$

parameter. Its critical value $\mu_{c}$, under which there exist a homoclinic solution, will be determined in a later procedure. Let

$$
x=x_{0}+\varepsilon x_{1}+\mathrm{L}=\sum_{n=0}^{+\infty} \varepsilon^{n} x_{n} .
$$

Expansions of $\mu_{c}$ and $f\left(\mu_{\mathrm{c}}, x, x\right)$ with respect to $\varepsilon$ can be expressed as:

$$
\begin{aligned}
& \mu_{c}=\mu_{c 0}+\varepsilon \mu_{c 1}+\mathrm{L}=\sum_{n=0}^{\infty} \varepsilon^{n} \mu_{c n}, \\
& f\left(\mu_{c}, x, x\right)=f\left(\mu_{c 0}, x_{0}, \&\right)+\varepsilon\left[\mu_{c 1} f,{ }_{x}\left(\mu_{c 0}, x_{0}, \&\right)\right. \\
& \left.+x_{1} f,{ }_{x}\left(\mu_{c 0}, x_{0}, \&\right)+\not \& f,{ }_{x<}\left(\mu_{c 0}, x_{0}, \&\right)\right] \\
& +\varepsilon^{2}\left[\mu_{c 2} f,_{\mu}\left(\mu_{c 0}, x_{0}, \&\right)+x_{2} f,_{x}\left(\mu_{c 0}, x_{0}, \text {, \& }\right)\right. \\
& +\notin f,_{x<}\left(\mu_{c 0}, x_{0}, \text { if }\right)+\mu_{c 1} x_{1} f,_{\mu x}\left(\mu_{c 0}, x_{0}, \text { if }\right)
\end{aligned}
$$

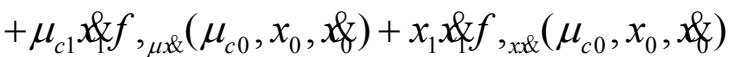

$$
\begin{aligned}
& +\frac{1}{2} \mu_{c 1}^{2} f,_{\mu \mu}\left(\mu_{c 0}, x_{0}, \&\right)+\frac{1}{2} x_{1}^{2} f,_{x x}\left(\mu_{c 0}, x_{0}, \&\right) \\
& \left.\left.+\frac{1}{2} \text { 这 } f, \mu_{c 0}, x_{0}, \text { \& }\right)\right]+\mathrm{L} \\
& =\left.\sum_{n=0}^{+\infty} \frac{\varepsilon^{n}}{n !} \frac{\left.\mathrm{d}^{n} f\left(\mu_{c}(\varepsilon), x(\varepsilon), x \notin \varepsilon\right)\right)}{\mathrm{d} \varepsilon^{n}}\right|_{\varepsilon=0}
\end{aligned}
$$

Where $f_{, \mu}=\partial f / \partial \mu, f_{, \mu x}=\partial^{2} f /(\partial \mu \partial x)$, etc. By substituting Eqs. (3), (4) and (5) into Eq. (2) and comparing coefficients of $\varepsilon$, we have

$$
\begin{aligned}
& \varepsilon^{0}:+c_{1} x_{0}+c_{2} x_{0}^{2}=0 ; \\
& \varepsilon^{1}:+\left(c_{1}+2 c_{2} x_{0}\right) x_{1}=f\left(\mu_{c 0}, x_{0}, \&\right) \text {; } \\
& \varepsilon^{2}:+\left(c_{1}+2 c_{2} x_{0}\right) x_{2}=\mu_{c 1} f,{ }_{\mu}\left(\mu_{c 0}, x_{0}, x_{f}\right)
\end{aligned}
$$

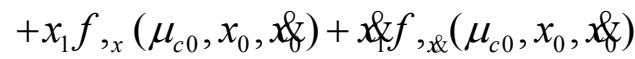

$$
\begin{aligned}
& -c_{2} x_{1}^{2} \text {; } \\
& \vdots \\
& \varepsilon^{n}:+\left(c_{1}+2 c_{2} x_{0}\right) x_{n} \text {, } \\
& \begin{aligned}
= & \left.\frac{1}{(n-1) !} \frac{\mathrm{d}^{n-1} f\left(\mu_{c}(\varepsilon), x(\varepsilon), x(\varepsilon)\right)}{\mathrm{d} \varepsilon^{n-1}}\right|_{\varepsilon=0} \\
& -c_{2} \sum_{i=1}^{n-1} x_{n-i} x_{i}, \quad(n \geq 2) ;
\end{aligned}
\end{aligned}
$$

in which $\mu$ is the homoclinic bifurcation 
Equation (6) has an exact analytical homoclinic solution in terms of hyperbolic functions [1]. The solution is

$x_{0}=a \operatorname{sech}^{2} \omega_{0} t+b$,

\& $=-2 a \omega_{0} \operatorname{sech}^{2} \omega_{0} t \tanh \omega_{0} t$.

$=2 a \omega_{0}^{2}\left(3 \tanh ^{2} \omega_{0} t-1\right) \operatorname{sech}^{2} \omega_{0} t$,

where

$\omega_{0}^{2}=\left|c_{1}\right| / 4$,

$a=3\left|c_{1}\right| / 2 c_{2}$,

$b=-\left(\left|c_{1}\right|+c_{1}\right) / 2 c_{2}$.

It is trivial to prove that Eqs. (10) and (11) satisfy Eq. (2). As [12]

$\operatorname{sech}( \pm \infty)=\tanh (0)=0$,

$\operatorname{sech}(0)=\tanh (+\infty)=1$,

$\tanh (-\infty)=-1$,

we have

$x_{0}(0)=a+b$,

$x_{0}( \pm \infty)=b$,

$\dot{x}_{0}( \pm \infty)=\ddot{x}_{0}( \pm \infty)=0$,

$\dot{x}_{0}(0)=0$.

Hence, Eqs. (14b,c) show that the homoclinic solution approaches the saddle point $(b, 0)$ in phase plane as time $t \rightarrow \pm \infty$.

Differentiating Eq. (6) with respect to $t$ leads to

$\dddot{x}_{0}+c_{1} \dot{x}_{0}+2 c_{2} x_{0} \dot{x}_{0}=0$.

It can be seen from Eq. (15) that $\dot{x}_{0}$ is a solution of the homogeneous part of Eq. (7). From the theory of linear differential equations, the particular solution of Eq. (7) can be expressed as:

$x_{1}=\dot{x}_{0} \int \frac{1}{\dot{x}_{0}^{2}}\left[\int \dot{x}_{0} f\left(\mu_{c 0}, x_{0}, \dot{x}_{0}\right) \mathrm{d} t\right] \mathrm{d} t$.

Since we are concerned only with the homoclinic solution which is independent of the initial conditions, the initial conditions and the homogeneous solution of $x 1$ are ignored. This practice is similar to that of the classical Lindstedt-Poincaré procedure for periodic solutions of autonomous oscillators [13].

As a homoclinic solution, $(x, \dot{x})$ approaches a saddle point in the phrase plane as time $t \rightarrow \pm \infty$. Thus, $x_{1}$ and $\dot{x}_{1}$ should be bounded as $t \rightarrow \pm \infty$. Mathematically,

$x_{1}( \pm \infty) \neq \pm \infty$,

$\dot{x}_{1}( \pm \infty) \neq \pm \infty$.

By multiplying both sides of Eq. (7) with $\dot{x}_{0}$ and integrating the equation from $-\infty$ to $+\infty$, we have

$$
\begin{aligned}
& \left.\left(\dot{x}_{0} \dot{x}_{1}-\ddot{x}_{0} x_{1}\right)\right|_{-\infty} ^{+\infty}+\int_{-\infty}^{+\infty}\left(\dddot{x}_{0}+c_{1} \dot{x}_{0}+2 c_{2} x_{0} \dot{x}_{0}\right) x_{1} \mathrm{~d} t . \\
& =\int_{-\infty}^{+\infty} \dot{x}_{0} f\left(\mu_{c 0}, x_{0}, \dot{x}_{0}\right) \mathrm{d} t
\end{aligned}
$$

With Eq. (15) invoked, the above equation is reduced to

$$
\left.\left(\dot{x}_{0} \dot{x}_{1}-\ddot{x}_{0} x_{1}\right)\right|_{-\infty} ^{+\infty}=\int_{-\infty}^{+\infty} \dot{x}_{0} f\left(\mu_{c 0}, x_{0}, \dot{x}_{0}\right) \mathrm{d} t .
$$

From Eqs. (14c) and (17), the left hand side of Eq. (19) must vanish, i.e.

$\int_{-\infty}^{+\infty} \dot{x}_{0} f\left(\mu_{c 0}, x_{0}, \dot{x}_{0}\right) \mathrm{d} t=0$.

Therefore, Eq. (20) is a necessary condition for Eq. $(17 \mathrm{a}, \mathrm{b})$. One can conveniently determine the critical value of $\mu 0$ by solving Eq. (20) and consequently avoid secular terms of $x 1$ in the later procedure. In an alternative yet more cumbersome way, one can ignore Eq. (20) but determine $\mu 0$ by eliminating the secular terms in $x 1$. This procedure will be illustrated more clearly by an example involving a generalized Liénard oscillator in the next section.

Multiplying $\dot{x}_{0}$ to Eq. (9) and integrating the equation from $-\infty$ to $+\infty$ give

$$
\begin{gathered}
\left.\left(\dot{x}_{0} \dot{x}_{2}-\ddot{x}_{0} x_{2}\right)\right|_{-\infty} ^{+\infty}+\int_{-\infty}^{+\infty}\left(\dddot{x}_{0}+c_{2} \dot{x}_{0}+2 c_{2} x_{0} \dot{x}_{0}\right) x_{2} \mathrm{~d} t \\
=\int_{-\infty}^{+\infty} \dot{x}_{0}\left[\mu_{c 1} f_{, \mu}\left(\mu_{c 0}, x_{0}, \dot{x}_{0}\right)+x_{1} f_{, x}\left(\mu_{c 0}, x_{0}, \dot{x}_{0}\right)\right.
\end{gathered}
$$




$$
\left.+\dot{x}_{1} f_{, \dot{x}}\left(\mu_{c 0}, x_{0}, \dot{x}_{0}\right)-c_{2} x_{1}^{2}\right] \mathrm{d} t
$$

By recalling Eq. (15), Eq. (21) is reduced as

$$
\begin{aligned}
& \left.\left(\dot{x}_{0} \dot{x}_{2}-\ddot{x}_{0} x_{2}\right)\right|_{-\infty} ^{+\infty}=\int_{-\infty}^{+\infty} \dot{x}_{0}\left[\mu_{c 1} f_{, \mu}\left(\mu_{c 0}, x_{0}, \dot{x}_{0}\right)\right. \\
& \left.\quad+x_{1} f_{, x}\left(\mu_{c 0}, x_{0}, \dot{x}_{0}\right)+\dot{x}_{1} f_{, \dot{x}}\left(\mu_{c 0}, x_{0}, \dot{x}_{0}\right)-c_{2} x_{1}^{2}\right] \mathrm{d} t .
\end{aligned}
$$

From Eq. (14c) and the conditions

$$
\begin{aligned}
& x_{2}( \pm \infty) \neq \pm \infty, \\
& \dot{x}_{2}( \pm \infty) \neq \pm \infty, \\
& \int_{-\infty}^{+\infty} \dot{x}_{0}\left[\mu_{c 1} f_{, \mu}\left(\mu_{c 0}, x_{0}, \dot{x}_{0}\right)+x_{1} f_{, x}\left(\mu_{c 0}, x_{0}, \dot{x}_{0}\right)\right. \\
& \left.\quad+\dot{x}_{1} f_{, \dot{x}}\left(\mu_{c 0}, x_{0}, \dot{x}_{0}\right)-c_{2} x_{1}^{2}\right] \mathrm{d} t=0 .
\end{aligned}
$$

Hence, the value of $\mu_{\mathrm{c} 1}$ under which there exists a homoclinic solution of Eq. (2) can be determined by Eq. (24). Consequently, one can eliminate the secular terms of the 2 nd order solution

$$
\begin{aligned}
& x_{2}=\dot{x}_{0} \int \frac{1}{\dot{x}_{0}^{2}}\left\{\int \dot { x } _ { 0 } \left[\mu_{c 1} f_{, \mu}\left(\mu_{c 0}, x_{0}, \dot{x}_{0}\right)\right.\right. \\
& \quad+x_{1} f_{, x}\left(\mu_{c 0}, x_{0}, \dot{x}_{0}\right)+\dot{x}_{1} f_{, \dot{x}}\left(\mu_{c 0}, x_{0}, \dot{x}_{0}\right) \\
& \left.\left.\quad-c_{2} x_{1}^{2}\right] \mathrm{~d} t\right\} \mathrm{d} t
\end{aligned}
$$

It is worth pointing out that Eq. (20) can also be derived to approximate $\mu_{\mathrm{c}}$ by the standard Melnikov method, Belhaq's technique combined with elliptic solutions $[10,11]$ or the hyperbolic perturbation method [1]. Using different methods, here we expand $\mu c$ in the form of Eq. (4) which can lead to a higher order approximation for critical value of $\mu c$. Furthermore, the explicit homoclinic solution can also be approximately constructed as the procedure is based on hyperbolic functions instead of periodic functions.

Similarly, one can determine the value of $\mu_{c}(n-1)$ by solving

$$
\begin{aligned}
& \int_{-\infty}^{+\infty} \dot{x}_{0}\left\{\left.\frac{1}{(n-1) !} \frac{\mathrm{d}^{n-1} f\left(\mu_{c}(\varepsilon), x(\varepsilon), \dot{x}(\varepsilon)\right)}{\mathrm{d} \varepsilon^{n-1}}\right|_{\varepsilon=0}\right. \\
& \left.-c_{2} \sum_{i=1}^{n-1} x_{n-i} x_{i}\right\} \mathrm{d} t=0 .
\end{aligned}
$$

Consequently, one can eliminate the secular terms of the n-th order solution

$$
x_{n}=\dot{x}_{0} \int \frac{1}{\dot{x}_{0}^{2}}\left\{\int \dot { x } _ { 0 } \left[\left.\frac{1}{(n-1) !} \frac{\mathrm{d}^{n-1} f\left(\mu_{c}(\varepsilon), x(\varepsilon), \dot{x}(\varepsilon)\right)}{\mathrm{d} \varepsilon^{n-1}}\right|_{\varepsilon=0}\right.\right.
$$

$$
\left.\left.-c_{2} \sum_{i=1}^{n-1} x_{n-i} x_{i}\right] \mathrm{~d} t\right\} \mathrm{d} t
$$

However, the procedure would be increasingly cumbersome as the solution order goes up. More importantly, the computational results show that the solution up to the order $\varepsilon x_{1}$ is fairly accurate even for the moderately large parameter $\varepsilon$ and the homoclinic bifurcation parameter curves predicted by the present method show a higher accuracy than those obtained by periodic solutions.

In the classical Lindstedt-Poincare procedure for periodic solution, one expands the nonlinear frequency with respect to $\varepsilon$ and determines the frequency by eliminating secular terms. In the present procedure for homoclinic solution, we expand the homoclinic bifurcation critical parameter $\mu_{c}$ with respect to $\varepsilon$ and similarly determines the critical parameter by eliminating the secular terms. In this light, the present method can be regarded as a hyperbolic Lindstedt-Poincaré method for homoclinic solutions of nonlinear oscillators.

\section{A study of the generalized Liénard oscillator}

As a sample application of the present method, the following generalized Liénard equation is considered:

$c_{1} x+c_{2} x^{2}=\varepsilon\left(\mu+\mu_{1} x-\mu_{2} x^{2}-\mu_{3} \& x\right)$

In other words,

$f(\mu, x, x)=x\left(\mu+\mu_{1} x-\mu_{2} x^{2}-\mu_{3} x\right)$,

in which $\mu_{1}$ and $\mu_{2}$ are constants whereas $\mu$ is 
considered as a homoclinic bifurcation parameter.

Let

$$
\begin{aligned}
I(t) & =\int \& f\left(\mu_{c 0}, x_{0}, \text {, \& }\right) \mathrm{d} t \\
& =\int \not \&\left(\mu_{c 0}+\mu_{1} x_{0}-\mu_{2} x_{0}^{2}-\mu_{3} \text { 媒 }\right) \mathrm{d} t .
\end{aligned}
$$

With $x_{0}$ and \& in Eqs. $(10 \mathrm{a}, \mathrm{b})$ substituted into Eq. (30), the latter equation becomes

$$
\begin{aligned}
I(t)= & 4 a^{2} \omega_{0}\left(A_{1}+A_{2} \operatorname{sech}^{2} \omega_{0} t\right. \\
& \left.+A_{3} \operatorname{sech}^{4} \omega_{0} t+A_{4} \operatorname{sech}^{6} \omega_{0} t\right) \tanh ^{3} \omega_{0} t \\
& +a A_{5}\left(1-3 \tanh ^{2} \omega_{0} t\right) \operatorname{sech}^{2} \omega_{0} t,
\end{aligned}
$$

where

$$
\begin{aligned}
A_{1}= & \frac{2}{15} \mu_{c 0}+\left(\frac{8}{105} a+\frac{2}{15} b\right) \mu_{1}-\left(\frac{16}{315} a^{2}+\frac{2}{15} b^{2}\right. \\
& \left.+\frac{16}{105} a b\right) \mu_{2} \\
A_{2}= & \frac{3}{2} A_{1}, \\
A_{3}= & \frac{1}{7} a \mu_{1}-\left(\frac{2}{7} a b+\frac{2}{21} a^{2}\right) \mu_{2} \\
A_{4}= & -\frac{1}{9} a^{2} \mu_{2}, \\
A_{5}= & -\frac{1}{6} a \mu_{3} .
\end{aligned}
$$

Thus, Eq. (20) or $\left.I(t)\right|_{-\infty} ^{+\infty}=0$ yields

$$
\begin{aligned}
4 a^{2} \omega_{0} & {\left[\left(A_{1}+A_{2} \operatorname{sech}^{2} \omega_{0} t+A_{3} \operatorname{sech}^{4} \omega_{0} t\right.\right.} \\
& \left.+A_{4} \operatorname{sech}^{6} \omega_{0} t\right) \tanh ^{3} \omega_{0} t \\
& \left.+A_{5} \omega_{0}\left(2-\frac{3}{2} \operatorname{sech}^{2} \omega_{0} t\right) \operatorname{sech}^{6} \omega_{0} t\right]\left.\right|_{-\infty} ^{+\infty} \\
= & 8 a^{2} \omega_{0} A_{1}=0 .
\end{aligned}
$$

For non-zero $a$ and $\omega_{0}$,

$$
A_{1}=0
$$

By virtue of Eq. (32b), the above equation implies

$$
A_{2}=0 \text {. }
$$

From the definition of $A_{1}$ in Eq. (32a),

$$
\mu_{c 0}=-\left(\frac{4}{7} a+b\right) \mu_{1}+\left(\frac{8}{21} a^{2}+b^{2}+\frac{8}{7} a b\right) \mu_{2} .
$$

From which the value of parameter $\mu_{\mathrm{c} 0}$ can be determined. Then, Eq. (16) becomes

$$
x_{1}=\dot{x}_{0} \int \frac{1}{\dot{x}_{0}^{2}} I(t) \mathrm{d} t
$$

After substituting Eq. (31) into Eq. (38), integration leads to

$$
\begin{aligned}
x_{1}= & a \operatorname{sech}^{2} \omega_{0} t\left\{-\frac{1}{\omega}\left[2 A_{3} \ln \left(\cosh \omega_{0} t\right)\right.\right. \\
& \left.\left.+A_{4} \tanh ^{2} \omega_{0} t\right] \tanh \omega_{0} t+A_{5}\left(1-3 \tanh ^{2} \omega_{0} t\right)\right\} \\
& +\frac{a}{\omega_{0}}\left[\left(\frac{1}{2} A_{1}+A_{2}\right) \operatorname{sech}^{2} \omega_{0} t \tanh \omega_{0} t\right. \\
& \left.-2 A_{2} \tanh \omega_{0} t-A_{1} \sinh 2 \omega_{0} t\right] .
\end{aligned}
$$

As $\sinh 2 \omega_{0} t$ tends to infinity as $t \rightarrow \infty$, $A_{1} \sinh 2 \omega_{0} t$ is a secular term for homoclinic solution. Hence, $A_{1}$ should vanish and this leads to Eq. (36). Consequently, the first order solution becomes

$$
\begin{aligned}
& x_{1}=a \operatorname{sech}^{2} \omega_{0} t\left\{-\frac{1}{\omega}\left[2 A_{3} \ln \left(\cosh \omega_{0} t\right)\right.\right. \\
& \left.\left.+A_{4} \tanh ^{2} \omega_{0} t\right] \tanh \omega_{0} t+A_{5}\left(1-3 \tanh ^{2} \omega_{0} t\right)\right\} .
\end{aligned}
$$

As mentioned in Section 2, one can substitute Eqs. (35) and (36) into Eq. (31) and then completes the integration of Eq. (38) to obtain Eq. (40). Then,

$$
\begin{aligned}
\dot{x}_{1} & =a\left[2\left(A_{4}-A_{3}\right)+\left(2 A_{3}-7 A_{4}\right) \operatorname{sech}^{2} \omega_{0} t\right. \\
& +5 A_{4} \operatorname{sech}^{4} \omega_{0} t+2 A_{3} \ln \left(\cosh \omega_{0} t\right)(2 \\
& \left.\left.-3 \operatorname{sech}^{2} \omega_{0} t\right)\right] \operatorname{sech}^{2} \omega_{0} t \\
& -4 A_{5} a \omega_{0} \operatorname{sech}^{2} \omega_{0} t \tanh \omega_{0} t\left(2-3 \tanh ^{2} \omega_{0} t\right) .
\end{aligned}
$$

To determine $\mu_{c 1}$, one inserts Eq. (29) into Eq. 
(24) and yields

$$
\begin{gathered}
\int_{-\infty}^{+\infty} \dot{x}_{0}\left[\mu_{c 1} \dot{x}_{0}+x_{1} \dot{x}_{0}\left(\mu_{1}-2 \mu_{2} x_{0}\right)+\dot{x}_{1}\left(\mu_{c 0}\right.\right. \\
\left.\left.+\mu_{1} x_{0}-\mu_{2} x_{0}^{2}-2 \mu_{3} \dot{x}_{0}\right)-c_{2} x_{1}^{2}\right] \mathrm{d} t=0 .
\end{gathered}
$$

By substituting $x_{0}$, \&,$x_{1}$ and if given in Eqs. (10), (12), (40) and (41) into Eq. (42), the latter can be integrated to be

$$
\begin{aligned}
& \frac{a^{2}}{\omega_{0}}\left\{B_{1}+B_{2} \operatorname{sech}^{2} \omega_{0} t+B_{3} \operatorname{sech}^{4} \omega_{0} t+B_{4} \operatorname{sech}^{6} \omega_{0} t\right. \\
& \quad+B_{5} \operatorname{sech}^{8} \omega_{0} t+B_{6} \operatorname{sech}^{10} \omega_{0} t \\
& \quad+B_{7}\left[\frac{1}{3} \operatorname{sech}^{6} \omega_{0} t \ln \left(\cosh \omega_{0} t\right)-\left(\frac{1}{15}-\frac{2}{21} \tanh ^{2} \omega_{0} t\right.\right. \\
& \left.\left.\left.\quad+\frac{1}{27} \tanh ^{4} \omega_{0} t\right) \tanh ^{2} \omega_{0} t\right] \tanh ^{2} \omega_{0} t\right\}\left.\tanh \omega_{0} t\right|_{-\infty} ^{+\infty} \\
& =\frac{2 a^{2}}{\omega_{0}}\left(B_{1}-\frac{8}{945} B_{7}\right)=0,
\end{aligned}
$$

where

$$
\begin{aligned}
B_{1} & =\frac{16}{21} \mu_{c 0} \omega_{0}^{2} A_{5}+\frac{8}{15} \mu_{c 1} \omega_{0}^{2}+\frac{16}{21} \mu_{1} A_{5} \omega_{0}^{2}\left(\frac{4}{5} a+b\right) \\
& -\frac{16}{21} \mu_{2} A_{5} \omega_{0}^{2}\left(\frac{8}{11} a^{2}+\frac{8}{5} a b+b^{2}\right)+\frac{128}{315} \mu_{3} a \omega_{0}^{2}\left(A_{3}\right. \\
& \left.+\frac{4}{11} A_{4}\right)-\frac{128}{3465} a c_{1} A_{4} A_{5} \\
B_{7} & =16 \mu_{3} a \omega_{0}^{2} A_{3}-8 a c_{1} A_{3} A_{5}
\end{aligned}
$$

and the coefficients $B_{2}$ to $B_{6}$ are listed in Appendix. For nonzero $a$, Eq. (43) implies

$$
B_{1}-\frac{8}{945} B_{7}=0
$$

By consolidating Eqs.(33),(37),(44) and (45), Eq. (46) yields

$$
\begin{aligned}
& \mu_{c 1}=-\frac{4}{14553} \mu_{3} a^{2}\left\{11 \mu_{1}\left(6-\frac{c_{1} a}{\omega_{0}^{2}}\right)+2 \mu_{2}[6(a-11 b)\right. \\
& \left.\left.+\frac{c_{1} a}{\omega_{0}^{2}}(6 a+11 b)\right]\right\} .
\end{aligned}
$$

Finally, the homoclinic solution of Eq. (28) can be obtained as

$$
\begin{aligned}
x= & a \operatorname{sech}^{2} \omega_{0} t+b \\
- & \varepsilon a \operatorname{sech}^{2} \omega_{0} t \frac{\tanh \omega_{0} t}{\omega_{0}}\left[2 A_{3} \ln \left(\cosh \omega_{0} t\right)+A_{4} \tanh ^{2} \omega_{0} t\right] \\
& \left.+A_{5}\left(3 \tanh ^{2} \omega_{0} t-1\right)\right\}+O\left(\varepsilon^{2}\right), \\
\&= & -2 a \omega_{0} \operatorname{sech}^{2} \omega_{0} t \tanh \omega_{0} t+\varepsilon a\left[2\left(A_{4}-A_{3}\right)\right. \\
& +\left(2 A_{3}-7 A_{4}\right) \operatorname{sech}^{2} \omega_{0} t+5 A_{4} \operatorname{sech}^{4} \omega_{0} t \\
& \left.+2 A_{3} \ln \left(\cosh \omega_{0} t\right)\left(2-3 \operatorname{sech}^{2} \omega_{0} t\right)\right] \\
& -4 \varepsilon A_{5} a \omega_{0}\left(2-3 \tanh ^{2} \omega_{0} t\right) \operatorname{sech}^{2} \omega_{0} t \tanh \omega_{0} t \\
& +O\left(\varepsilon^{2}\right), \\
\mu_{c}= & -\left(\frac{4}{7} a+b\right) \mu_{1}+\left(\frac{8}{21} a^{2}+b^{2}+\frac{8}{7} a b\right) \mu_{2} \\
& -\varepsilon \frac{4}{14553} \mu_{3} a^{2}\left\{11 \mu_{1}\left(6-\frac{c_{1} a}{\omega_{0}^{2}}\right)+2 \mu_{2}[6(a-11 b)\right. \\
& \left.\left.+\frac{c_{1} a}{\omega_{0}^{2}}(6 a+11 b)\right]\right\}+O\left(\varepsilon^{2}\right) .
\end{aligned}
$$

\section{Examples}

In this section, four examples would be presented to assess the efficacy and accuracy of the present method.

Example 1. Consider the following equation:

$x-0.4 x^{2}=\varepsilon(\mu-0.2 x) x$,

which is a case of the oscillator in Eq. (28) with $c_{1}=1, c_{2}=-0.4, \mu_{1}=-0.2, \mu_{2}=0$ and $\mu_{3}=0$. With $\varepsilon=1$, the example has been studied by both the standard Melnikov method and Belhaq's technique combined with trigonometric solution to second order by multiple scales method [9]. The homoclinic bifurcation curves of the equation in the $\omega-\mu$ plane were investigated by trigonometric multiple scales method. Here, $\omega=\sqrt{c_{1}}$ denotes the linear frequency of the linear generating equation. 
By using the present method and from Eqs. (12), $a=-3.75, b=2.5$ and $\omega=0.5$. Through Eq. (33), $A_{3}=3 / 28, A_{4}=0$ and $A_{5}=0$. The homoclinic solution of Eq. (51) is then solved to be

$x=-\frac{15}{4} \operatorname{sech}^{2} \frac{t}{2}+\frac{5}{2}+\frac{9}{56} \varepsilon \ln \left(\cosh \frac{t}{2}\right) \operatorname{sech}^{2} \frac{t}{2} \tanh \frac{t}{2}+O\left(\varepsilon^{2}\right)$, $x=\frac{15}{4} \operatorname{sech}^{2} \frac{t}{2} \tanh \frac{t}{2}-\frac{27}{112} \varepsilon \ln \left(\cosh \frac{t}{2}\right) \operatorname{sech}^{2} \frac{t}{2} \tanh ^{2} \frac{t}{2}$ $+\frac{9}{112} \varepsilon\left[\ln \left(\cosh \frac{t}{2}\right)+\tanh ^{2} \omega t\right] \operatorname{sech}^{2} \omega t+O\left(\varepsilon^{2}\right)$

With $\varepsilon=1, \mu_{c}=0.071428$ can be obtained from Eq. (50) or by the standard Melnikov method [9]. The homoclinic orbit is shown in Figure 1. The homoclinic bifurcation curves in the $\varepsilon-\mu_{\mathrm{c}}$ plane and in the $\omega-\mu$ plane are plotted in Figure 2 and Figure 3, respectively.
Fig. 1 Homoclinic orbit and limit cycle at $\mu_{\mathrm{c}}$ for Eq. (51) with $\varepsilon=1.0$.

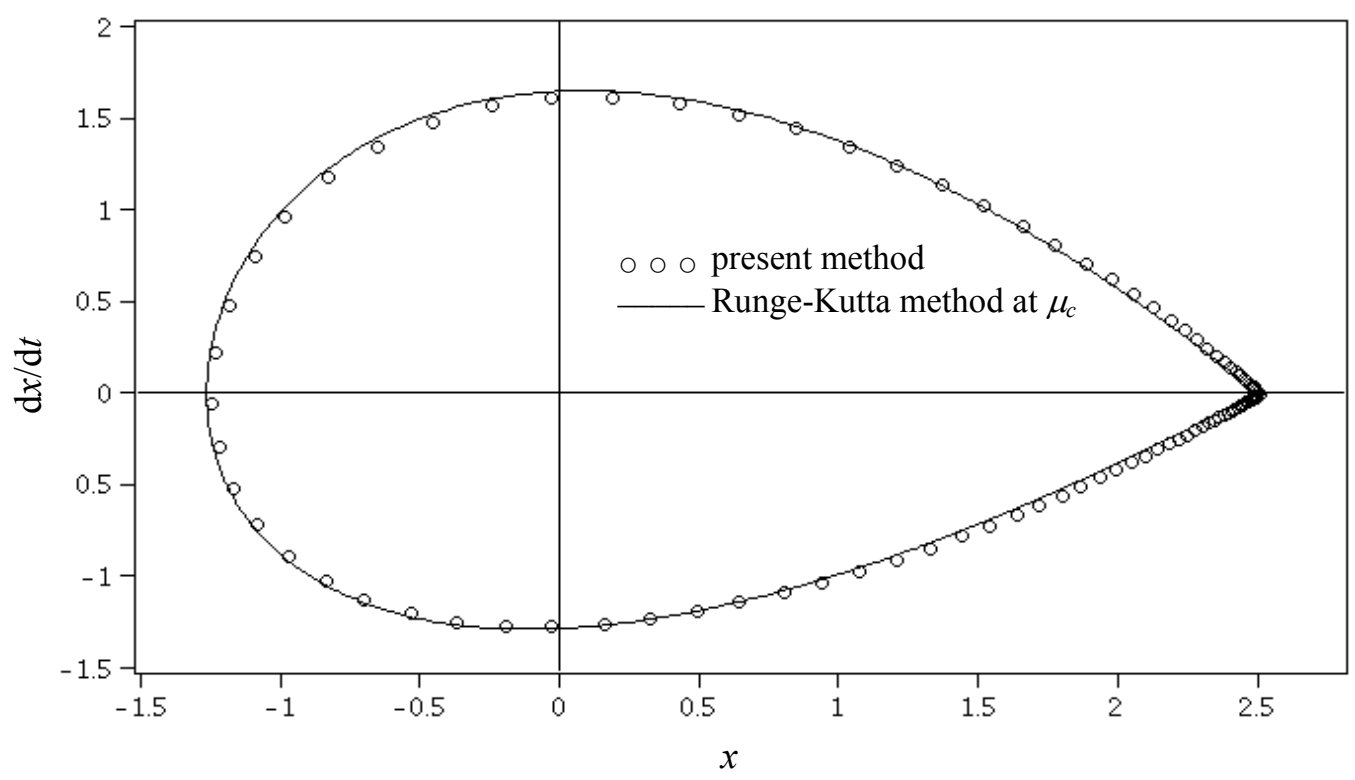

Fig. 2

Homoclinic bifurcation curves in the $\varepsilon-\mu$ plane for Eq. (51).

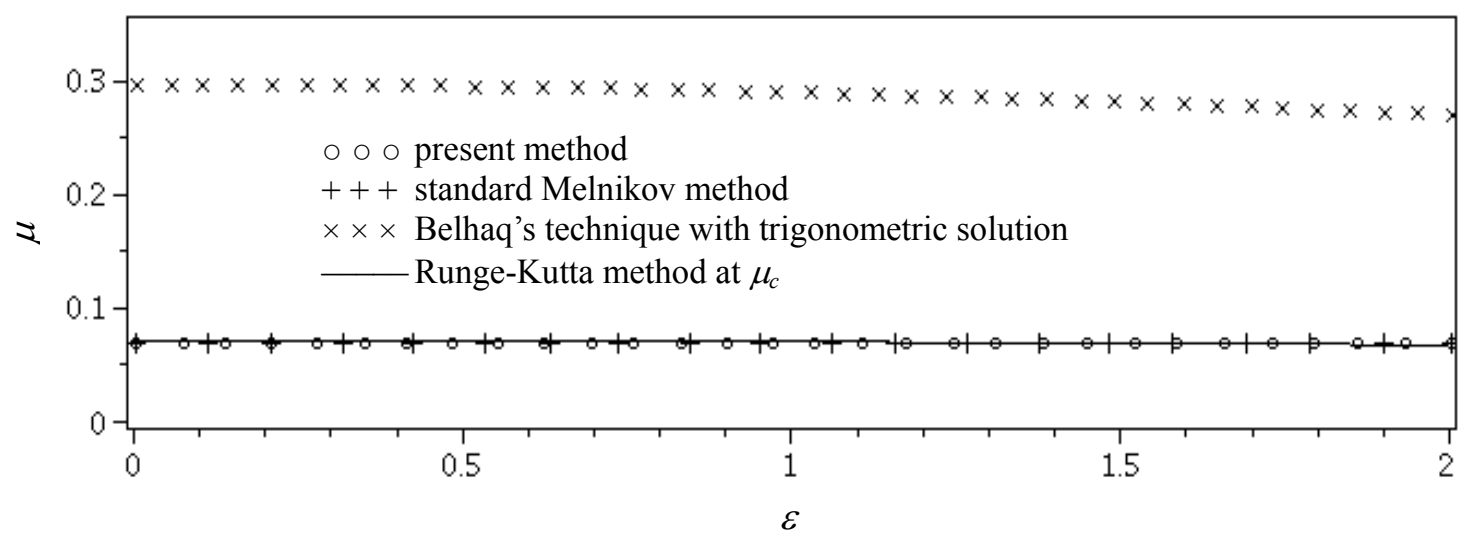

Fig. 3

Homoclinic bifurcation curves in the $\omega-\mu$ plane for Eq. (51) with $\varepsilon=1.0$.

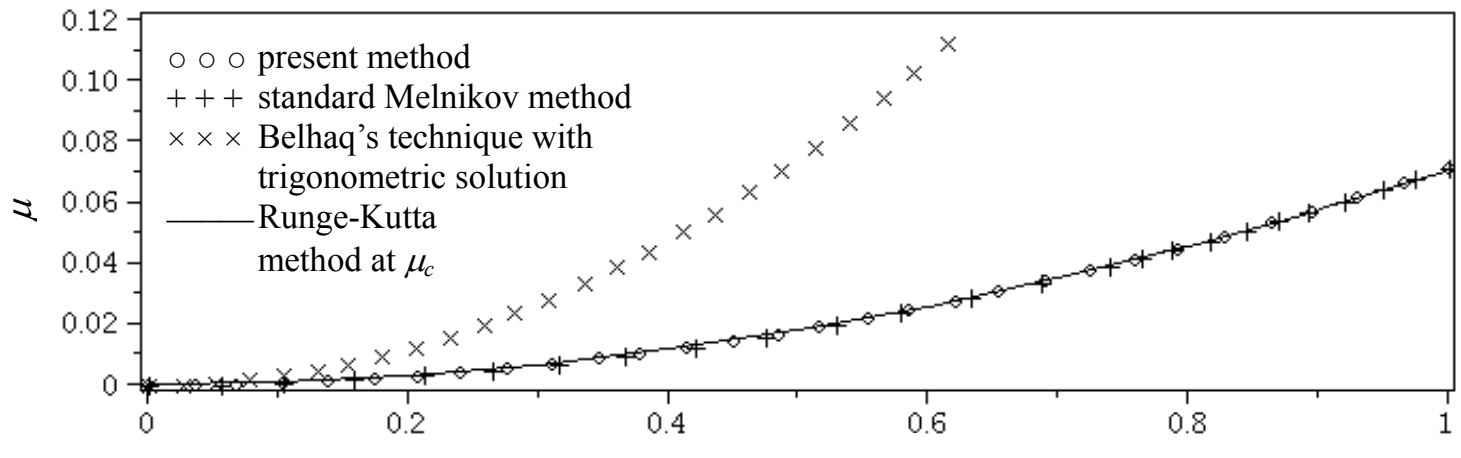


In this paper, the procedure of using Runge-Kutta integration method to determine the value of $\mu_{c}$ of the homoclinic orbit follows that of Merkin and Needham [14]. Numerical integration is conducted for a given value of $\varepsilon$ starting from a value of $\mu$ at which there is a limit cycle. It is repeated for increasing or reducing $\mu$ until a value of $\mu$ is reached such that there is no limit cycle. By successfully reducing the interval of $\mu$ within which a limit cycle disappears, a critical value $\mu_{c}$ can be identified such that a limit cycle can be found at $\mu=\mu_{c}$ but not at $\mu=\mu_{c} \pm \Delta$ where $\Delta$ is a small preset tolerance. Here, $\Delta$ is taken to be $10^{-6}$. Using this trial-and-error approach, $\mu_{c}=0.070493$. The value is close to but slightly lower than 0.071 428 obtained by the present method.

Example 2. Consider the following equation:

$0.5 x-2 x^{2}=\varepsilon(\mu+2 x+0.2 x)$,

which is a case of Eq. (28) with $c_{1}=1 c_{2}=3, \mu_{1}=$ $2.5, \mu_{2}=0$ and $\mu_{3}=1$. From Eq. (12), $a=0.5, b=$ $-1 / 3$ and $\omega=0.5$. Through Eq. (33), $A_{3}=5 / 28, A_{4}$ $=0$ and $A_{5}=-1 / 12$. The homoclinic solution of Eq. (51) is solved to be

$$
\begin{aligned}
x= & \frac{1}{2} \operatorname{sech}^{2} \frac{t}{2}-\frac{1}{3}+\frac{\varepsilon}{2}\left\{\frac{5}{7} \ln \left(\cosh \frac{t}{2}\right) \tanh \frac{t}{2}\right. \\
& \left.-\frac{1}{12}\left(1-3 \tanh ^{2} \frac{t}{2}\right)\right\} \operatorname{sech}^{2} \frac{t}{2}+O\left(\varepsilon^{2}\right), \\
\& \&= & -\frac{1}{2} \operatorname{sech}^{2} \frac{t}{2} \tanh \frac{t}{2}+\frac{5 \varepsilon}{28}\left[-1+\operatorname{sech}^{2} \omega t\right. \\
& \left.+\left(2-3 \operatorname{sech}^{2} \frac{t}{2}\right) \ln \left(\cosh \frac{t}{2}\right)\right] \\
& +\frac{1}{12}\left(2-3 \tanh ^{2} \omega t\right) \operatorname{sech}^{2} \omega t \tanh \omega t+O\left(\varepsilon^{2}\right) .
\end{aligned}
$$

With $\varepsilon=0.9$, one obtains $\mu_{c}=0.114560$ by Runge-Kutta method, 0.119047 by standard Melnikov method and 0.112244 from Eq. (50). The homoclinic orbit is shown in Figure 4. The homoclinic bifurcation curves in the $\varepsilon$ - $\mu$ plane and in the $\omega-\mu$ plane are plotted in Figure 5 and Figure
6 , respectively. In the figures, the predictions of the standard Melnikov method, Belhaq's technique [9] and Runge-Kutta method are also shown.

Example 3. Consider the following equation:

$2 x-x^{2}=\varepsilon\left(\mu+x-x^{2}+0.1 x\right) x$,

which is a case of Eq. (28) with $c_{1}=2, c_{2}=-1, \mu_{1}$ $=1, \mu_{2}=-1$ and $\mu_{3}=-0.1$. From Eq. (12), $a=-3, b$ $=2$ and $\omega=1 / \sqrt{2}$. Through Eq. (33), $A_{3}=3 / 7$, $A_{4}=-1$ and $A_{5}=-1 / 20$. The homoclinic solution of Eq. (53) is solved to be

$$
\begin{aligned}
x= & -3 \operatorname{sech}^{2} \frac{t}{\sqrt{2}}+2+3 \varepsilon \operatorname{sech}^{2} \frac{t}{\sqrt{2}}\left\{\left[\frac{6}{7} \sqrt{2} \ln \left(\cosh \frac{t}{\sqrt{2}}\right)\right.\right. \\
& \left.\left.-\tanh ^{2} \frac{t}{\sqrt{2}}-\frac{3}{20} \tanh \frac{t}{\sqrt{2}}\right] \tanh \frac{t}{\sqrt{2}}+\frac{1}{20}\right\}+O\left(\varepsilon^{2}\right), \\
x= & 3 \sqrt{2} \operatorname{sech}^{2} \frac{t}{\sqrt{2}} \tanh \frac{t}{\sqrt{2}}-3 \varepsilon\left[-\frac{20}{7}+\frac{55}{7} \operatorname{sech}^{2} \frac{t}{\sqrt{2}}\right. \\
& \left.-5 \operatorname{sech}^{4} \frac{t}{\sqrt{2}}+\frac{6}{7}\left(2-3 \operatorname{sech}^{2} \frac{t}{\sqrt{2}}\right) \ln \left(\cosh \frac{t}{\sqrt{2}}\right)\right] \\
& -\frac{3 \sqrt{2}}{10}\left(2-3 \tanh ^{2} \frac{t}{\sqrt{2}}\right) \operatorname{sech}^{2} \frac{t}{\sqrt{2}} \tanh \frac{t}{\sqrt{2}}+O\left(\varepsilon^{2}\right) .
\end{aligned}
$$

With $\varepsilon=1.0$, one obtains $\mu_{\mathrm{c}}=0.237149$ by Runge-Kutta method and 0.236734 from Eq. (50). The homoclinic orbit for is shown in Figure 7 whilst The homoclinic bifurcation curve in the $\varepsilon-\mu$ plane is plotted in Figure 8. In the figures, comparisons are also made with Runge-Kutta method.

Example 4. Consider the following equation:

$x-2 x^{2}=\varepsilon\left(\mu+x+3 x^{2}-0.5 x\right) x \&$,

which is a case of Eq. (28) with $c_{1}=-1 c_{2}=-2, \mu_{1}=1$, $\mu_{2}=-3$ and $\mu_{3}=0.5$. From Eqs. (12), $a=-3 / 4, b=0$, $\omega=1 / 2$. Through Eq. (33), $A_{3}=3 / 56, A_{4}=3 / 16$ and $A_{5}=1 / 16$. Then the homoclinic solution of Eq. (54) is solved to be 


$$
\begin{aligned}
x & =-\frac{3}{4} \operatorname{sech}^{2} \frac{t}{2}-\frac{3}{4} \varepsilon \operatorname{sech}^{2} \frac{t}{2}\left\{\frac { 3 } { 2 } \left[\frac{1}{7} \ln \left(\cosh \frac{t}{2}\right)\right.\right. \\
& \left.\left.+\frac{1}{4} \tanh ^{2} \frac{t}{2}\right] \tanh \frac{t}{2}+\frac{1}{16}\left(3 \tanh ^{2} \frac{t}{2}-1\right)\right\}+O\left(\varepsilon^{2}\right) \\
x & =\frac{3}{4} \operatorname{sech}^{2} \frac{t}{2} \tanh \frac{t}{2}-\frac{3}{64} \varepsilon\left[\frac{30}{7}-\frac{135}{7} \operatorname{sech}^{2} \frac{t}{2}\right. \\
& \left.+15 \operatorname{sech}^{4} \frac{t}{2}+6\left(2-3 \operatorname{sech}^{2} \frac{t}{2}\right) \ln \left(\cosh \frac{t}{2}\right)\right]
\end{aligned}
$$

$$
-\frac{3}{32}\left(2-3 \tanh ^{2} \frac{t}{2}\right) \operatorname{sech}^{2} \frac{t}{2} \tanh \frac{t}{2}+O\left(\varepsilon^{2}\right) .
$$

With $\varepsilon=0.9$, one obtains $\mu_{\mathrm{c}}=-0.232285$ by Runge-Kutta method and -0.224095 from Eq. (50). The homoclinic orbit for the case is shown in Figure 9. The homoclinic bifurcation curve in the $\varepsilon$ - $\mu$ plane is plotted in Figure 10. Comparisons are also made with Runge-Kutta method.
Fig. 4 Homoclinic orbit and limit cycle at $\mu_{c}$ for Eq.(52) with $\varepsilon=0.9$.

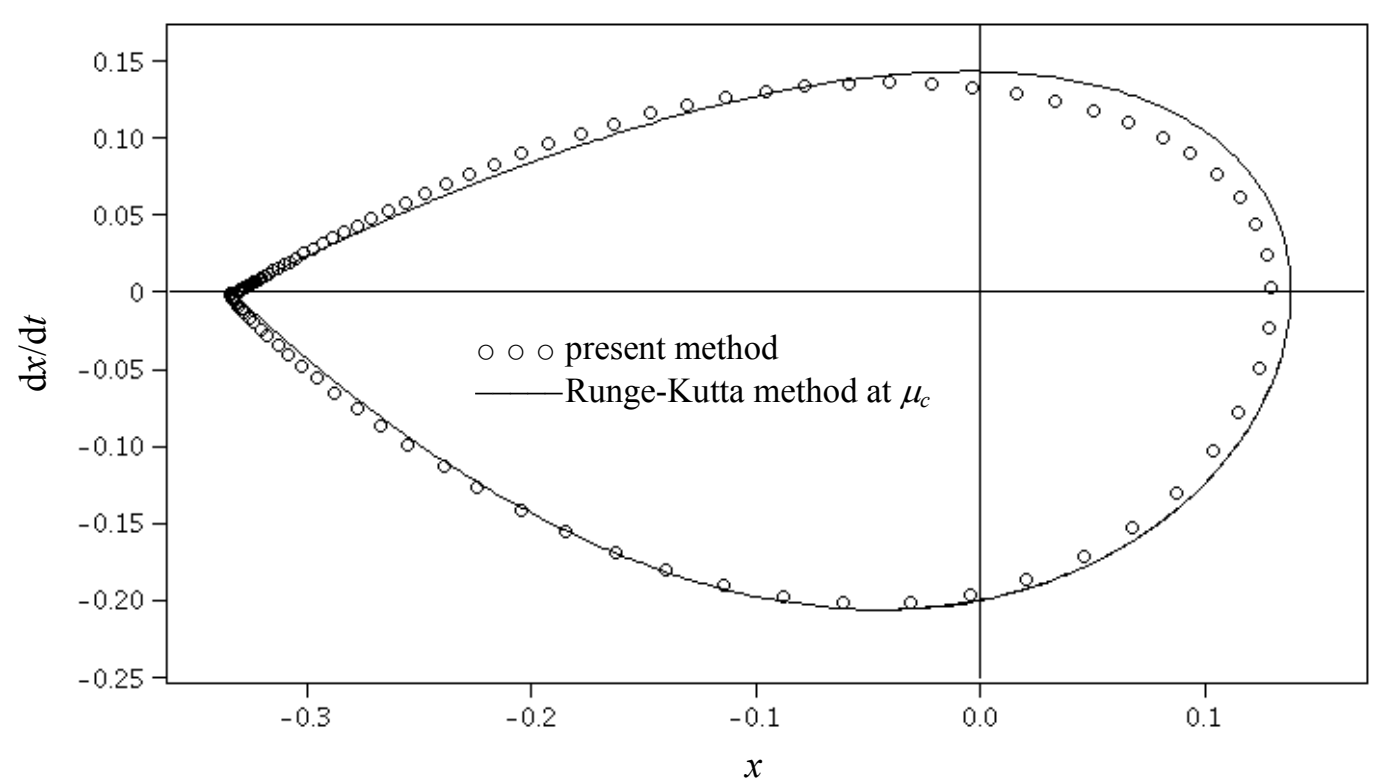

Fig. 5

Homoclinic Bifurcation curves in the $\varepsilon-\mu$ plane for Eq. (52).

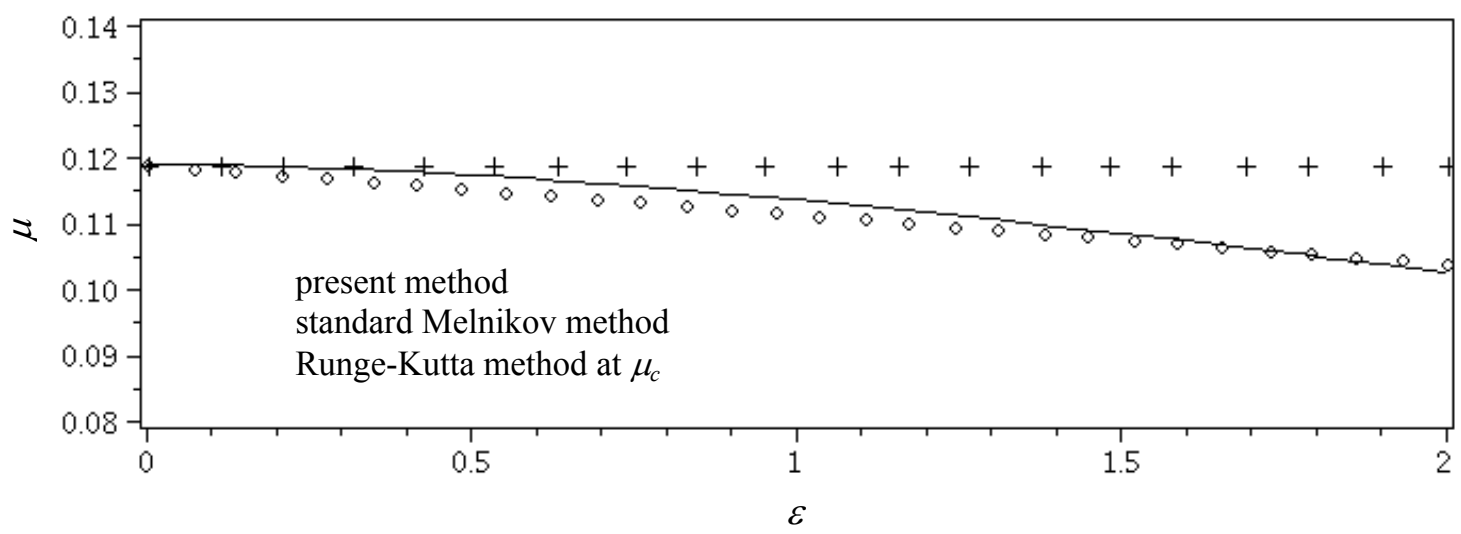

Fig. 6

Homoclinic Bifurcation curves in the $\omega-\mu$ plane for Eq. (52) with $\varepsilon=0.9$.

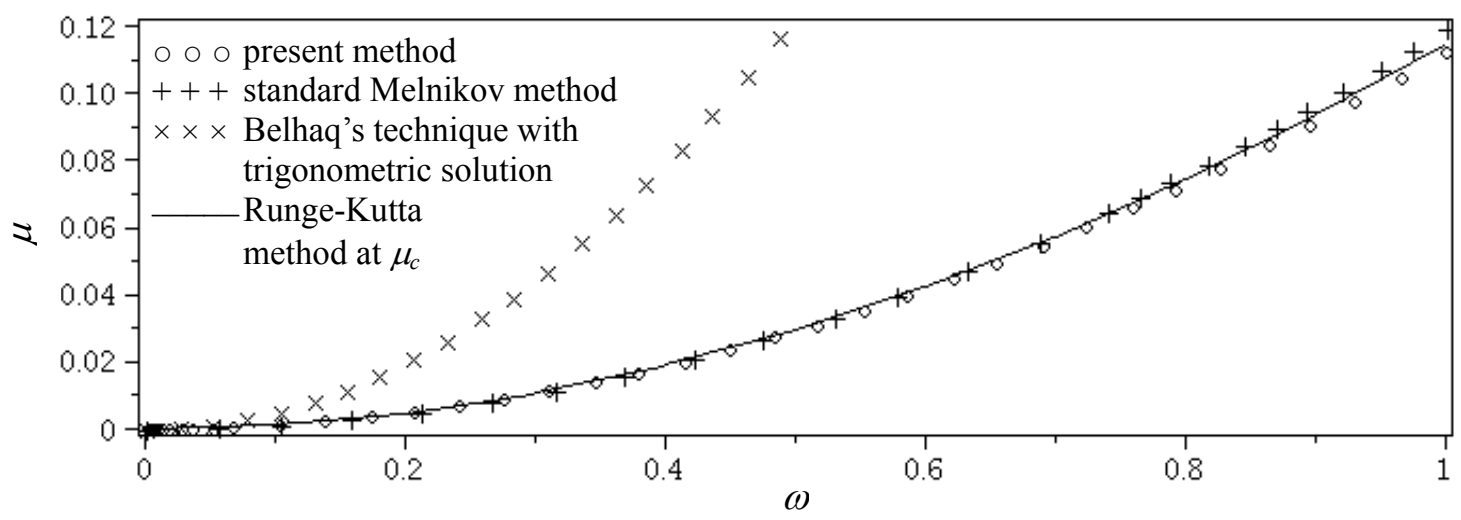


Fig. 7 Homoclinic orbit and limit cycle at $\mu_{c}$ for Eq. (53) with $\varepsilon=1.0$.

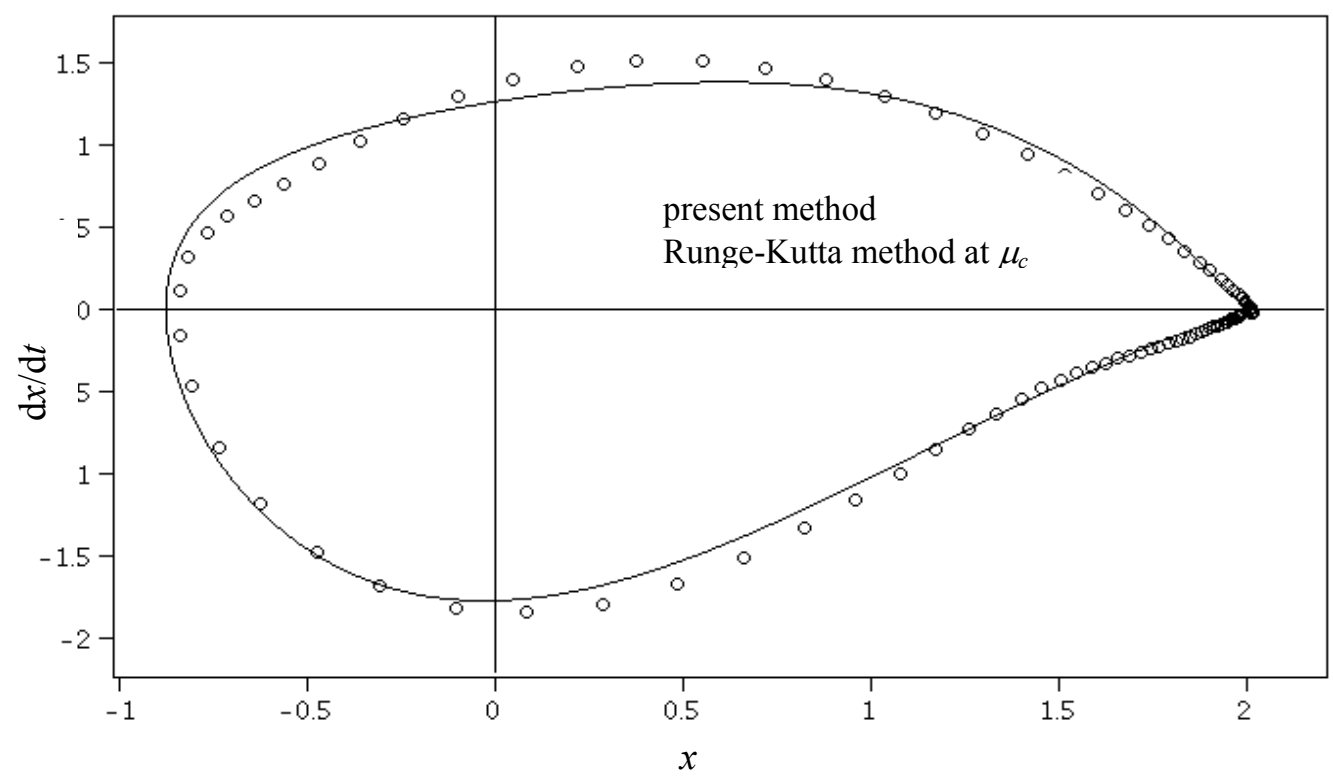

Fig. 8

Homoclinic bifurcation curves in the $\varepsilon$ - $\mu$ plane for Eq. (53).

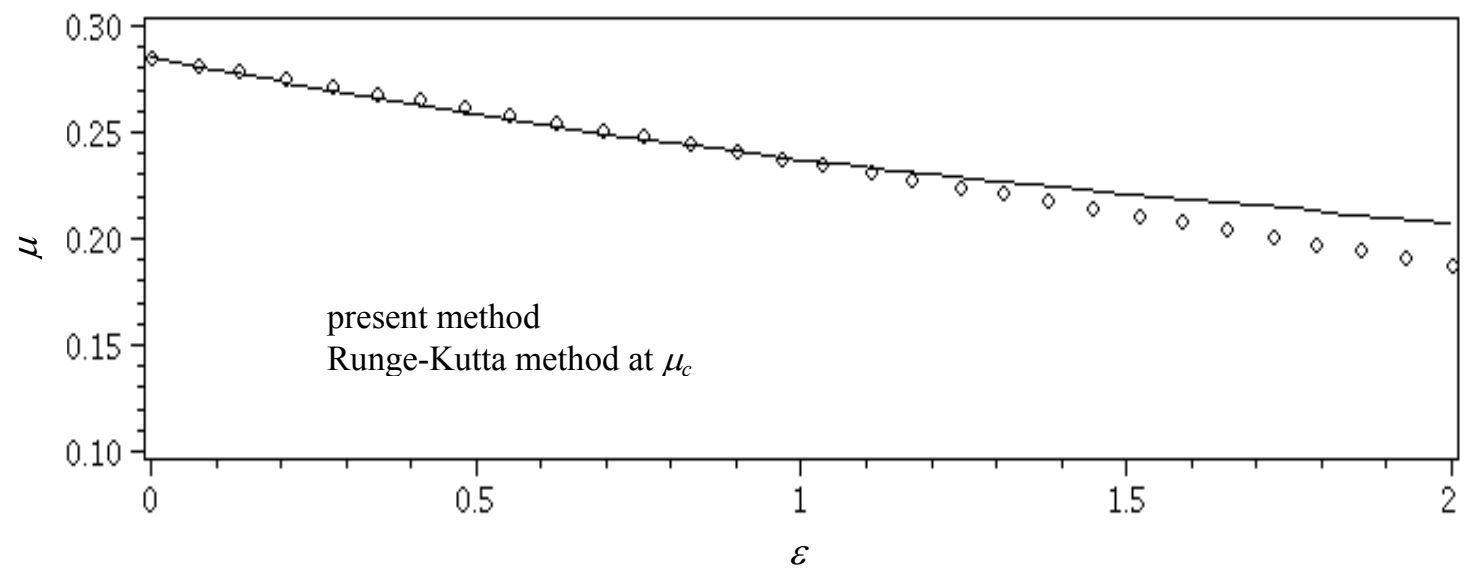

Fig 9. Homoclinic orbit and limit cycle at $\mu_{c}$ for Eq. (54) with $\varepsilon=0.9$.

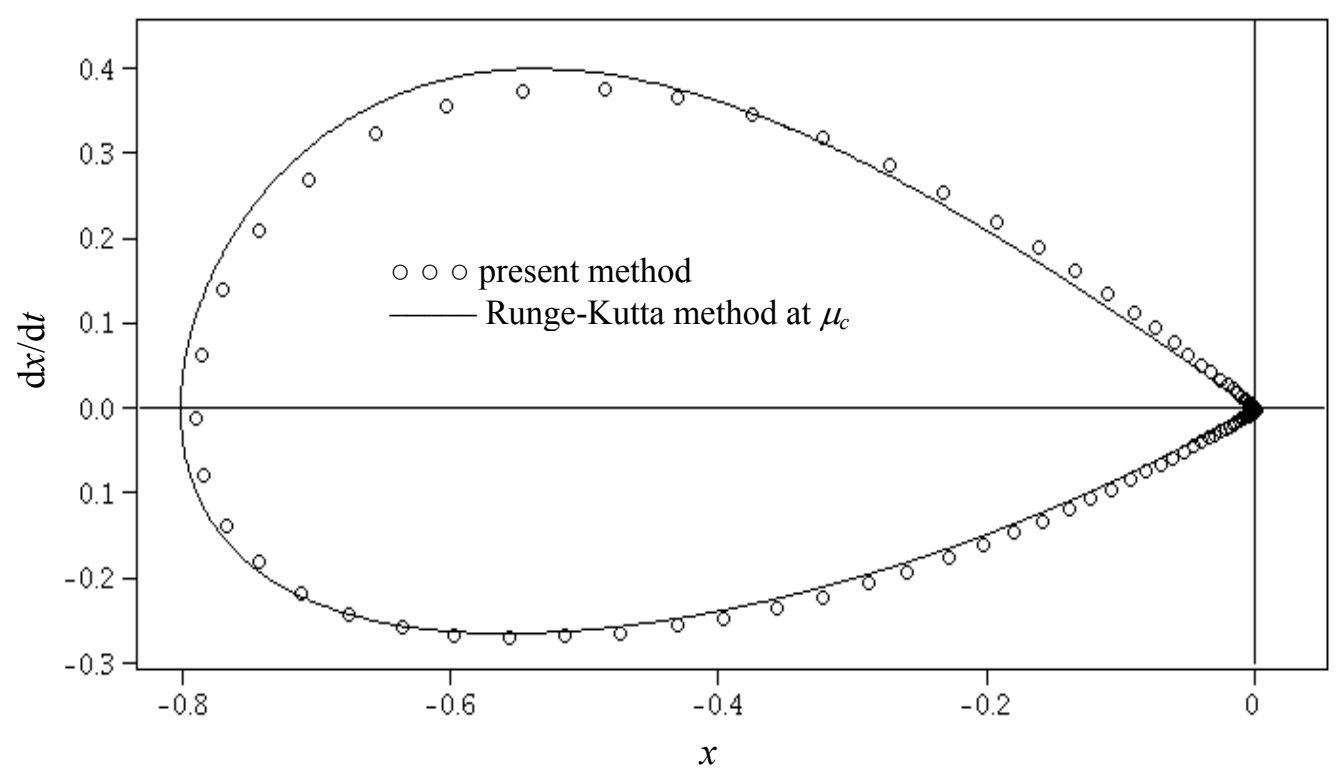


Fig. 10

Homoclinic

bifurcation

curves in the

$\varepsilon$ - $\mu$ plane

for Eq. (54).

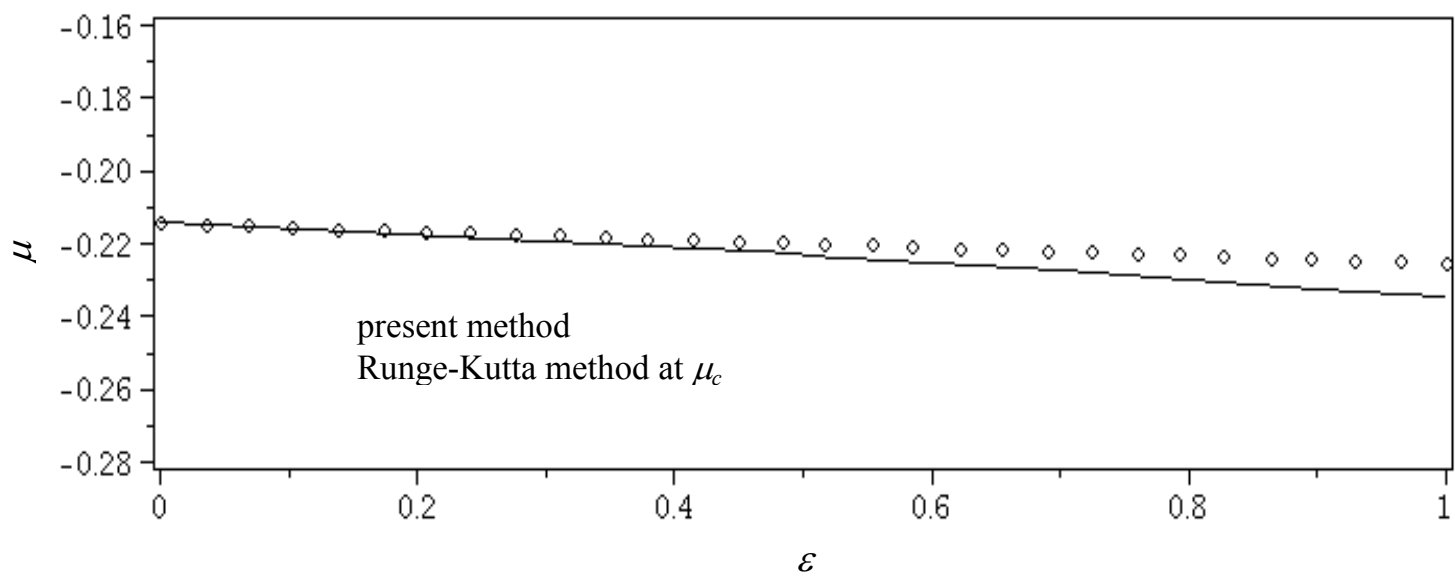

It can be seen from Figure 1, 2, 3, 4, 5, 6, 7, 8, 9 and 10 that the present predictions for the four examples are in good agreement with those obtained by the Runge-Kutta method even if $\varepsilon$ is moderately large. The predicted homoclinic orbits are close to those obtained by Runge-Kutta method at the critical value $\mu_{c}$. Figures $2,3,5$ and 6 also indicate that the present method is more accurate in analytically predicting the homoclinic bifurcation than the standard Melnikov method and Belhaq's technique with trigonometric solution. Meanwhile, it is worth noting that the $\mu_{c}$ value predicted by Runge-Kutta method is only a close approximation of the critical value of $\mu$ and the result of Runge-Kutta method at $\mu_{c}$ is still a limit cycle which is a periodic solution. At the exact critical value of $\mu$, a homoclinic orbit which is a solution with infinite period should be obtained.

\section{Conclusions}

(1) The hyperbolic Lindstedt-Poincaré method presented in this paper is an efficient method to construct approximate homoclinic solutions for certain nonlinear autonomous oscillators.

(2) The critical value of $\mu$, under which there exists a homoclinic solution of the nonlinear autonomous oscillator, can be approximately determined by the present perturbation procedure.

(3) Typical examples show that good accuracy of the homoclinic orbits obtained by present method even for moderately large value of $\varepsilon$.

(4) The present hyperbolic Lindstedt-Poincaré method can be generalized to determine heteroclinic solutions for certain strongly nonlinear autonomous oscillators.

\section{References}

1. Chen S.H., Chen Y.Y., Sze K.Y.: A hyperbolic perturbation method for determining homoclinic solution of certain strongly nonlinear autonomous oscillators. Journal of Sound and Vibration. 322, 381-392 (2009)

2. Wang, Z.H., Hu, H.Y.: A modified averaging scheme with application to the secondary Hopf bifurcation of a delayed van der Pol oscillator. Acta Mech. Sin. 24(4), 449-454 (2008)

3. Gan, C.B., He, S.M.: Studies on structural safety in stochasticallyexcited Duffing oscillator with double potential wells. Acta Mech. Sin. 23(5), 577-583 (2007)

4. Xu Z., Chan H. S. Y., Chung K. W.: Separatrices and limit cycles of strongly nonlinear oscillators by the perturbation-incremental method. Nonlinear Dynamics. 11, 213-233 (1996)

5. Chan H. S. Y., Chung K. W., Xu Z.: Stability and bifurcations of limit cycles by the perturbation-incremental method. Journal of Sound and Vibration. 206, 589-604 (1997)

6. Chen S. H., Chan J. K. W., Leung A. Y. T.: A perturbation method for the calculation of semi-stable limit cycles of strongly nonlinear 
oscillators. Communication in Numerical Methods in Engineering. 16, 301-313 (2000)

7. Zhang Y. M., Lu Q. S.: Homoclinic bifurcation of strongly nonlinear oscillators by frequency-incremental method, Communications in Nonlinear Science and Numerical Simulation. 8(1), 1-7 (2003)

8. Zhang Q., Wang W., Li W.: Heteroclinic bifurcations of strongly nonlinear oscillator, Chinese Physics Letters. 25(5), 1905-1907 (2008)

9. Belhaq M.: Predicting homoclinic bifurcations in planar autonomous systems. Nonlinear Dynamics. 18, 303-310 (1999)

10. Belhaq M., Lakrad F.: Prediction of homoclinic bifurcation: the elliptic averaging method. Chaos Solitons \& Fractals. 11, 2251-2258 (2000)

11. Belhaq M., Fiedler B., Lakrad F.: Homoclinic connections in strongly self-excited nonlinear oscillators: the Melnikov function and the elliptic Lindstedt-Poincaré method. Nonlinear Dynamics. 23, 67-86 (2000)

12. Abramowitz M. and Stegun I. A. (Editors).: Handbook of Mathematical Functions. New York, Dover (1972)

13. Nayfeh A. H.: Introduction to Perturbation Techniques. New York, Wiley (1981)

14. Merkin J.H., Needham D.J.: On infinite period bifurcations with an application to roll waves. Acta Mechanica. 60, 1-16 (1986)

\section{Appendix}

The coefficients $B_{2}, B_{3}, B_{4}, B_{5}$ and $B_{6}$ in Eq. (43) are:

$$
B_{2}=\frac{8}{21} \mu_{c 0} \omega_{0}^{2} A_{5}+\frac{4}{15} \mu_{c 1} \omega_{0}^{2}+\frac{8}{21} \mu_{1} A_{5} \omega_{0}^{2}\left(\frac{4}{5} a+b\right)
$$

$$
\begin{aligned}
& -\frac{8}{21} \mu_{2} A_{5} \omega_{0}^{2}\left(\frac{104}{165} a^{2}+\frac{8}{5} a b+b^{2}\right) \\
& +\frac{64}{315} \mu_{3} a \omega_{0}^{2}\left(A_{3}+\frac{4}{11} A_{4}\right)-\frac{64}{3465} a c_{1} A_{4} A_{5}, \\
B_{3}= & \frac{16}{7} \mu_{c 0} \omega_{0}^{2} A_{5}-\frac{4}{5} \mu_{c 1} \omega_{0}^{2}+\frac{8}{7} \mu_{1} A_{5} \omega_{0}^{2}\left(\frac{1}{5} a+2 b\right) \\
& -\frac{16}{7} \mu_{2} A_{5} \omega_{0}^{2}\left(\frac{13}{165} a^{2}+\frac{1}{5} a b+b^{2}\right) \\
& +\frac{16}{105} \mu_{3} a \omega_{0}^{2}\left(A_{3}+\frac{4}{11} A_{4}\right)-\frac{16}{1155} a c_{1} A_{4} A_{5}, \\
B_{4}= & -\frac{24}{7} \mu_{c 0} \omega_{0}^{2} A_{5}+\frac{4}{7} \mu_{1} A_{5} \omega_{0}^{2}(5 a-6 b) \\
& -\frac{8}{7} \mu_{2} A_{5} \omega_{0}^{2}\left(\frac{13}{99} a^{2}+\frac{8}{3} a b-3 b^{2}\right) \\
& -\frac{40}{63} \mu_{3} a \omega_{0}^{2}\left(4 A_{3}-\frac{47}{11} A_{4}\right)+\frac{916}{693} a c_{1} A_{4} A_{5}, \\
B_{5}= & -4 \mu_{1} A_{5} a \omega_{0}^{2}-\frac{16}{3} \mu_{2} A_{5} \omega_{0}^{2}\left(\frac{7}{33} a^{2}-a b\right) \\
& +\frac{16}{9} \mu_{3} a \omega_{0}^{2}\left(A_{3}-\frac{41}{11} A_{4}\right)-\frac{232}{99} a c_{1} A_{4} A_{5}, \\
B_{6}= & \frac{24}{11} \mu_{2} A_{5} a^{2} \omega_{0}^{2}+\frac{40}{11} \mu_{3} A_{4} a \omega_{0}^{2} .
\end{aligned}
$$

